\title{
Transarterial Embolization Using Pingyangmycin-Lipiodol Emulsion (PLE) for Treatment of Focal Nodular Hyperplasia of the Liver and 1-Year Follow-Up: A Case Report and Literature Review
}

\author{
Junyao Qiu1, Song Wen²*, Laishu Luo², Ping Chen1, Wuhua Guo ${ }^{{ }^{*}}$ \\ ${ }^{1}$ Department of Gastroenterology, Second Affiliated Hospital, Nanchang University, Nanchang, China \\ ${ }^{2}$ Department of Radiology, Second Affiliated Hospital, Nanchang University, Nanchang, China \\ Email: " Songwen81@163.com, ${ }^{*}$ guowuhuadoctor@126.com
}

Received 20 November 2014; revised 21 December 2014; accepted 2 January 2015

Copyright (C) 2015 by authors and Scientific Research Publishing Inc.

This work is licensed under the Creative Commons Attribution International License (CC BY).

http://creativecommons.org/licenses/by/4.0/

\section{(c) (i) Open Access}

\begin{abstract}
Focal nodular hyperplasia (FNH) is a common benign hepatic tumor which is often treated nonoperatively until it appears apparent symptoms such as pain and upper abdominal distension. We report here a case of trans-catheter arterial embolization (TAE) using pingyangmycin-lipiodol emulsion (PLE) for blocking a fatal bleeding originated from percutaneous biopsy as well as for FNH treatment. After embolization by means of the mixture of PLE and particles of gelfoam, the bleeding was stopped and the FNH was apparently shrinked 1 year later. TAE using PLE may be considered as a safe and effective method for the treatment of FNH.
\end{abstract}

Keywords

Embolization, Focal Nodular Hyperplasia, Pingyangmycin

\section{Introduction}

Focal nodular hyperplasia (FNH), a benign hepatic tumor, is often difficult to distinguish from other hepatic lesions such as hemangioma, focal hepatic steatosis, hepatoma, liver metastasis [1]. Although FNH is a benign lesion that is typically managed nonoperatively in adults, most children with FNH currently undergo resection "Corresponding authors. 
because of symptoms, increasing size, or inability to confidently rule out malignancy [2]. Surgical resection including laparoscopic hepatectomy is the commonly used management for FNH [1]. FNH is a hypervascular nodule, which is usually fed by a single end-artery with no intratumoral arteriovenous shunting or parasitic blood supply. So it may be a good choice for the patients who must be mandated intervention by means of arterial embolization. Recently, Huang et al. demonstrated that transarterial embolization (TAE) using pingyangmycin-lipiodol emulsion (PLE) and polyvinyl alcohol is safe and effective for the treatment of FNH in 4 patients [3]. However, transarterial embolization using PLE and polyvinyl alcohol for treatment of fatal hemorrhage of FNH after imaging-guided percutaneous biopsy is not reported.

Here we report a case of FNH, which suffered from hypovolemic shock due to fatal bleeding after CT-guided percutaneous biopsy and underwent TAE for bleeding control as well as FNH treatment effectively.

\section{Case Report}

A 65-year-old man suffered from unexplained melena, and a mass in right lobe of liver and left kidney respectively by abdominal ultrasonography. Upper gastrointestinal endoscope confirmed an ulcer in gastric antrum, and histopathologic examination demonstrated the diagnosis of chronic superficial gastritis. CT scan helped to confirm the presences of an $8.1 \times 7.5$ centimeters lesion in right lobe of liver and a maximal diameter $6.5 \mathrm{~cm}$ lesion in left kidney (Figure 1(A) and Figure 1(B)). Contrast-enhanced CT showed the lesion in right lobe of liver had abundance of blood supply (Figure 1(A) and Figure 1(C)), and some low density organization (Figure 1(D)). The level alpha-fetoprotein was $13.9 \mathrm{ng} / \mathrm{ml}$, and routine laboratory investigations and liver function were all within normal range.

In order to distinguish the lesion from hepatocellular carcinoma and liver metastasis because of the drastically different therapeutic approach, a percutaneous biopsy was performed under computed tomography, and $18 \mathrm{G} \times$ $150 \mathrm{~mm}$ needle (Precisa, Italy) was employed to capture tumor organization. In the procedure of twice puncture, CT scan showed that hemoperitoneum in the patient increased rapidly (Figure 2(A) and Figure 2(B)). Almost 5
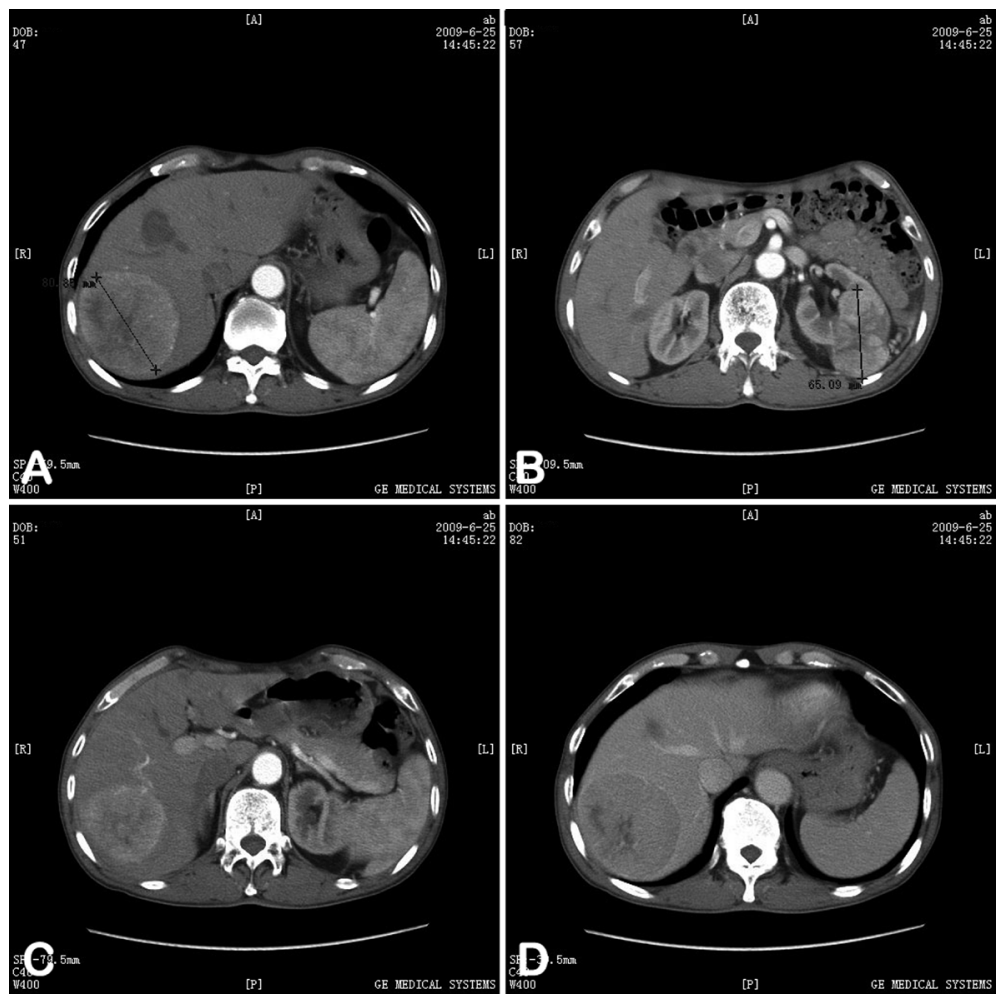

Figure 1. CT imaging of FNH. A mass in the right lobe of liver (A) and a mass in the left kidney (B) were detected in Gd-DTPA-enhanced CT imaging. The mass in right lobe of liver showed abundance of blood supply (C) and low density organization in the central of mass (D). 
minutes after liver puncture, the patient developed signs and symptoms of shock including pallor, hypotension (87/50 mmHg), tachycardia (107 beats per minute), cold, clammy extremities and altered mental state in CT department. An urgent trans-catheter arterial embolization was decided to undergo to control bleeding of liver.

After informed consent was obtained from his family members, digital subtraction angiography (DSA, OEC9800 C-arm X-ray system, GE, USA) of hepatic artery was performed through right femoral artery using a 6F, $40 \mathrm{~cm}$ Flexor Check-Flo sheath and 5F RH catheter (Cook Medical, Bloomington, IN). Common hepatic arteriography demonstrated the hypervascular right lobe lesion supplied by the right hepatic artery branches (Figure 3(A)) and the bleeding sign was not detected. After the 5F catheter was correctly positioned into proper hepatic artery, the mixture of pingyangmycin (Qilu Pharmaceutical Co., Ltd., China) $12 \mathrm{mg}$ and lipiodol (Guerbet Co., France) $15 \mathrm{ml}$ was infused into the artery under the guidance of X-ray. Then particles of gelfoam $(2 \times 2$ $\mathrm{mm}$ ) were infused until the blood supply of lesion disappeared (Figure 3(B)). And then an 8F pigtail drainage catheter (Cook) was percutaneous inserted into right lower abdomen to drain the abdominal hemoperitoneum. In the process of embolization, 2 units of erythrocyte were transfused to improve the anemia.

Histopathologic examination of hepatic lesion demonstrated focal nodular hyperplasia (Figure 4). Because of the diagnosis of hepatic benign tumor, the patient was performed a radical nephrectomy 10 days after TAE. The histopathologic examination of the left renal mass was suprarenal epithelioma. The patient's general gradually recovered after radical nephrectomy, and did not show any immediate or delayed postembolization complications. Follow-up CT at 1.5 month demonstrated shrinkage and no vascularity to the lesion (Figure 5A) and at 1.4-year follow-up showed that the volume of FNH reduced more than $65 \%(5.2 \times 4.1 \mathrm{~cm}$, Figure 5(B)). Furthermore, routine laboratory investigations, alpha-fetoprotein, liver function were all within normal range.
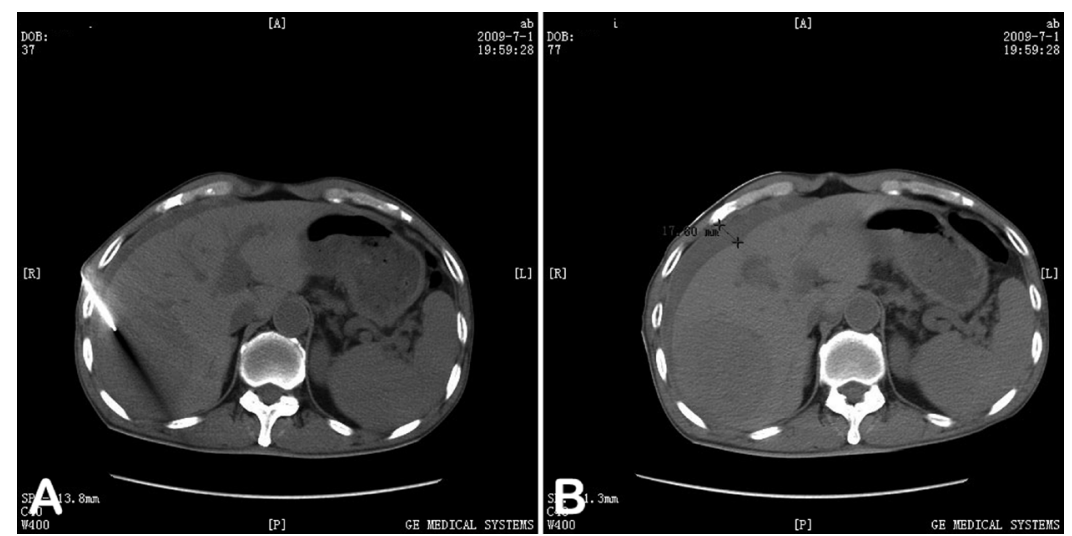

Figure 2. CT imaging of hemorrhage of FNH. CT scan showed that hemoperitoneum increased rapidly in the process (A) and after percutaneous biopsy (B).

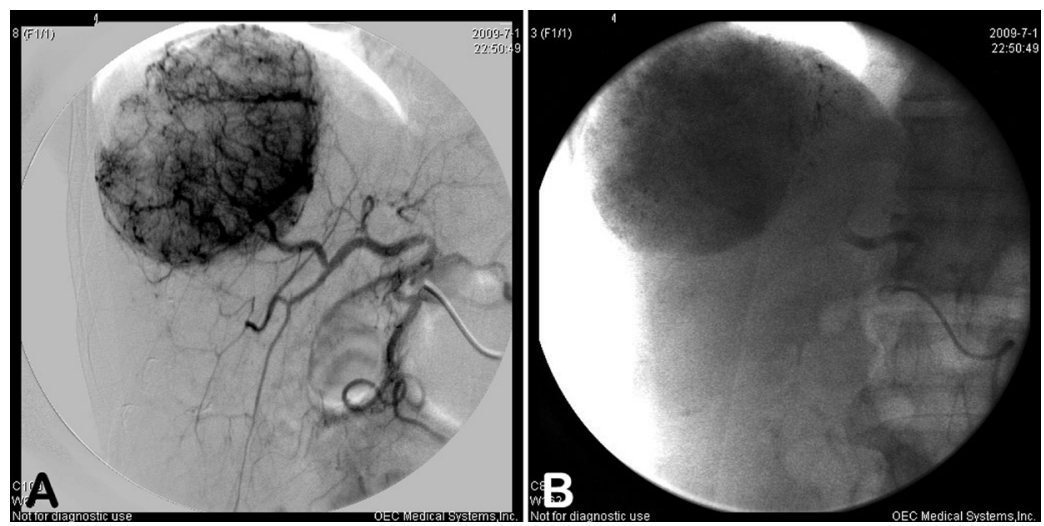

Figure 3. DSA imaging of FNH. Arteriography demonstrated the hypervascular right lobe lesion supplied by the right hepatic artery branches (A). (B) indicated that the blood supply of mass disappeared after infusing the mixture of PLE and particles of gelfoam. 


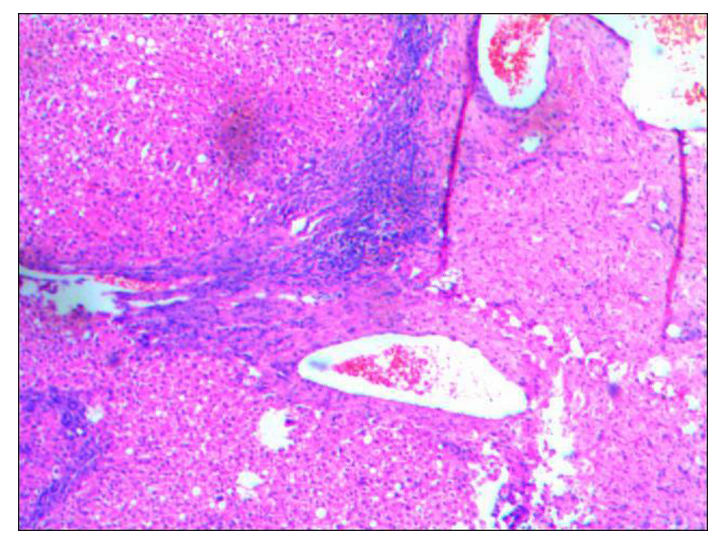

Figure 4. Histopathologic examination of hepatic lesion. Hematoxylin and eosin stain demonstrated focal nodular hyperplasia (magnification 100×).
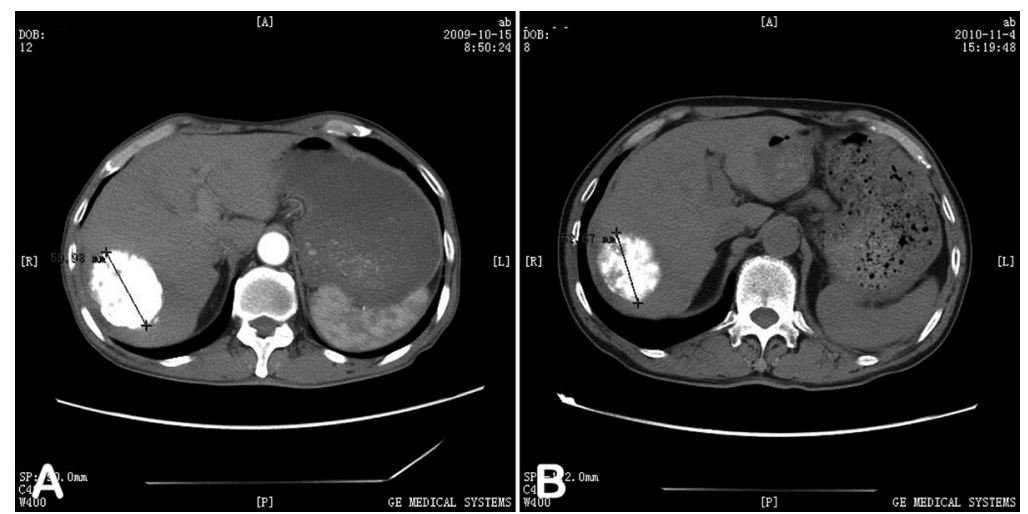

Figure 5. CT imaging of FNH after TAE using PLE. CT images showed that the volume of FNH reduced 1.5-month (A) and 1.4-year (B) after TAE using PLE gradually.

\section{Discussion}

FNH is one of the most common benign neoplasms of liver and accounts for $8 \%$ of all primary hepatic tumors [1] [4]. Image-guided needle-biopsy is necessary when the differential diagnosis from other nodular lesions of liver is difficult [5]. Although image-guided needle-biopsy is an effective and minimally invasive mean to differentiate FNH from other hepatic lesions, it has the apparent shortcoming such as hemorrhage. Super-selective TAE of the bleeding vessel of FNH is an effective, minimally invasive and relatively easy procedure in needle-biopsy induced hemorrhage, with high rate of success and immediate benefits [6] [7].

Recently, Huang first reported that treatment of FNH in 4 patient using PLE and polyvinyl alcohol. Follow-up examination by CT or MRI ranging from 12 to 42 months revealed complete resolution in 2 cases and an obvious tumor volume decrease in the other 2 cases [3]. our case provides further evidence supporting that TAE using PLE may considered as a safe and effective method for the treatment of FNH.

Pingyangmycin (PYM) has been found to reduce DNA synthesis of cancer cells and cut off DNA chain, shows a strong damage function to tumor endothelial cells [8], which proved effective and safe for treatment of cheilitis granulomatosa [9] and gigantic cavernous hemangioma of liver [10]. With the emulsifying function of PLE, PYM can be accumulated in the FNH at a high concentration and slow released. For treating FNH of PYM in current case, it may rely on its ability to destroy the endothelial cells, resulting in the formation of microthrombi in sinuses and causing atrophy and fibrosis of the tumor mass. In addition, PLE does not cause any severe damage within normal vessels because it can be washed out rapidly. Last but not the least, the main severe complication caused by PYM is pulmonary fibrosis. However, no pulmonary fibrosis has been observed in the present series of patients after follow-up. The maximum dosage of PYM used in current study was much 
lower than the toxic dose level. It may have been the main reason for preventing this dosage-dependent complication.

\section{Conclusion}

In conclusion, we report a case of TAE using PLE for stopping a fatal bleeding originated from percutaneous biopsy as well as FNH treatment effectively. Future studies are needed to evaluate the long term safety and effectiveness of PLE in the treatment of FNH through TAE.

\section{Acknowledgements}

This work was supported by a grant from National Natural Science Foundation of China (\#81401460 and \#91029720).

\section{References}

[1] Navarro, A.P., Gomez, D., Lamb, C.M., Brooks, A. and Cameron, I.C. (2014) Focal Nodular Hyperplasia: A Review of Current Indications for and Outcomes of Hepatic Resection. HPB (Oxford), 16, 503-511. http://dx.doi.org/10.1111/hpb.12169

[2] Cha, D.I., Yoo, S.Y., Kim, J.H., Jeon, T.Y. and Eo, H. (2014) Clinical and Imaging Features of Focal Nodular Hyperplasia in Children. American Journal of Roentgenology, 202, 960-965. http://dx.doi.org/10.2214/AJR.13.11856

[3] Huang, D., Chen, Y., Zeng, Q., Zhao, J., Wu, R., et al. (2011) Transarterial Embolization Using Pingyangmycin Lipiodol Emulsion and Polyvinyl Alcohol for the Treatment of Focal Nodular Hyperplasia of the Liver. Hepatogastroenterology, 58, 1736-1741. http://dx.doi.org/10.5754/hge11174

[4] Donadon, M., Di Tommaso, L., Roncalli, M. and Torzilli, G. (2013) Multiple Focal Nodular Hyperplasias Induced by Oxaliplatin-Based Chemotherapy. World Journal of Hepatology, 5, 340-344.

[5] Pupulim, L.F., Vullierme, M.P., Paradis, V., Valla, D., Terraz, S., et al. (2013) Congenital Portosystemic Shunts Associated with Liver Tumours. Clinical Radiology, 68, e362-e369. http://dx.doi.org/10.1016/j.crad.2013.01.024

[6] Ronot, M. and Vilgrain, V. (2014) Imaging of Benign Hepatocellular Lesions: Current Concepts and Recent Updates. Clinics and Research in Hepatology and Gastroenterology, 38, 681-688.

[7] Carrafiello, G., Lagana, D., Ianniello, A., Craparo, G., Recaldini, C., et al. (2007) Bleeding after Percutaneous Radio Frequency Ablation: Successful Treatment with Transcatheter Embolization. European Journal of Radiology, 61, 351355. http://dx.doi.org/10.1016/j.ejrad.2006.10.001

[8] Chen, W.L., Huang, Z.Q., Zhang, D.M. and Chai, Q. (2010) Percutaneous Sclerotherapy of Massive Venous Malformations of the Face and Neck Using Fibrin Glue Combined with OK-432 and Pingyangmycin. Head Neck, 32, 467-472.

[9] Gu, L., Huang, D.Y., Fu, C.J., Wang, Z.L., Liu, Y., et al. (2013) Intralesional Injection of Pingyangmycin plus Corticosteroids May Be an Effective Treatment for Cheilitis Granulomatosa. Medical Hypotheses, 81, 729-730. http://dx.doi.org/10.1016/j.mehy.2013.07.037

[10] Zeng, Q., Li, Y., Chen, Y., Ouyang, Y., He, X., et al. (2004) Gigantic Cavernous Hemangioma of the Liver Treated by Intra-Arterial Embolization with Pingyangmycin-Lipiodol Emulsion: A Multi-Center Study. CardioVascular and Interventional Radiology, 27, 481-485. http://dx.doi.org/10.1007/s00270-003-2754-2 
Scientific Research Publishing (SCIRP) is one of the largest Open Access journal publishers. It is currently publishing more than 200 open access, online, peer-reviewed journals covering a wide range of academic disciplines. SCIRP serves the worldwide academic communities and contributes to the progress and application of science with its publication.

Other selected journals from SCIRP are listed as below. Submit your manuscript to us via either submit@scirp.org or Online Submission Portal.
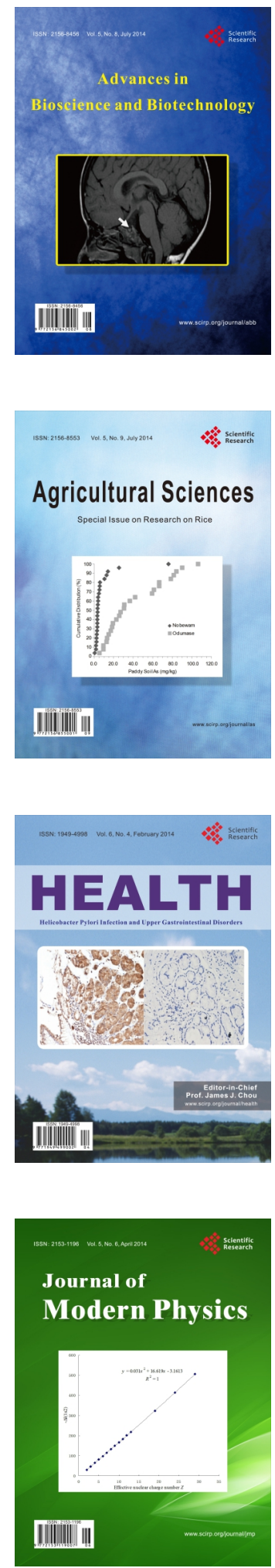
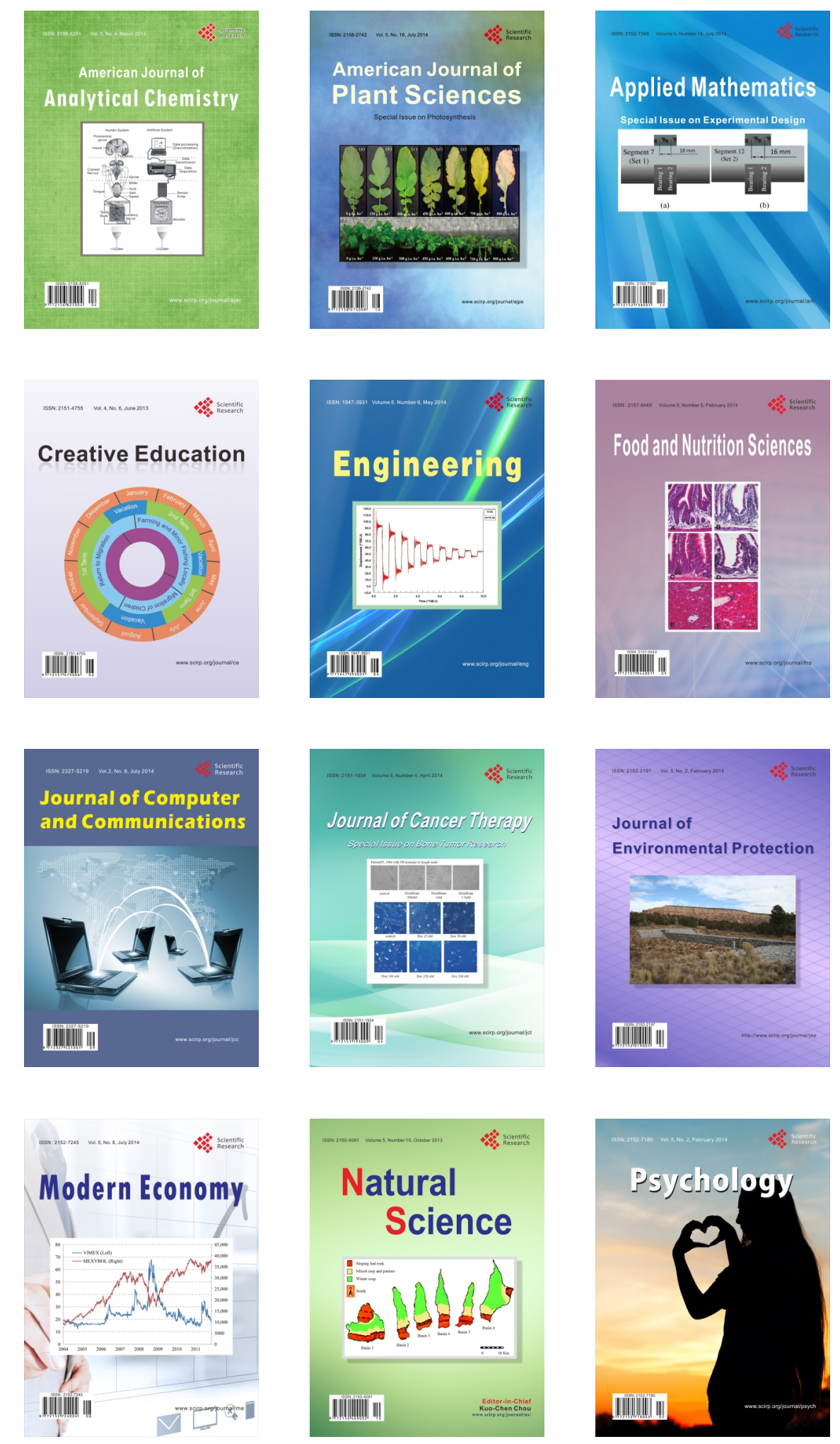